\title{
VIBRATION EVALUATION MODEL ON THE WHEELCHAIR TRANSPORTING APPARATUS
}

\author{
Yoshiyuki MATSUOKA
}

Keio University, 3 - 14 -1, Hiyoshi, Kohokuku, Yokohama, Kanagawa, 223 -8522, Japan

\begin{abstract}
Vehicles modified with a wheelchair transporting apparatus (WTA) are generally used for improving wheelchair users' mobility. However, in designing the apparatus, consideration for passengers' discomfort and fatigue is not sufficient. As a countermeasure of this problem, both vibration characteristics of the passenger-wheelchair system and passengers' sensitivity need to be studied. In previous study, authors constructed a vibration simulation model to predict vibration on passengers' body from floor vibration. The objectives of the present study are to clarify the relationship between vibration and comfort, and to propose a method to support designing a WTA by the findings of this study.

First, we carried out a vibration experiment with a vibration generator that vibrated the passenger-wheelchair system and measured floor vibration, vibration on the human body, and subjective evaluation.

Second, we discussed experimental results. The result of riding comfort measurements proved that the characteristics of sensitivity to vibration differ in two frequency ranges: from $2 \mathrm{~Hz}$ to $6 \mathrm{~Hz}$ and from $6 \mathrm{~Hz}$ to $12 \mathrm{~Hz}$. Therefore we divided the results into two sections for these two frequency ranges.

Relationship between floor vibration and riding comfort was examined. We carried out the multiple regression analysis and deduced the formulas that express the value of riding comfort by the acceleration and frequency of the vibration on the floor. Next, relationship between body vibration and riding comfort was examined. From the result of correlation analysis and multiple regression analysis, vertical vibration on the upper torso is found to be the most closely related to the riding comfort. Regression formulas that express the value of riding comfort by the acceleration and frequency of the vertical vibration on the upper torso were deduced.

Finally, we proposed a vibration evaluation model that can predict riding comfort from the vertical vibration on the upper torso, which will be valuable in designing a WTA.
\end{abstract}

Key Word: Evaluation of vibration, Riding comfort, Wheelchair

\section{INTRODUCTION}

Vehicles modified with a wheelchair transporting apparatus (WTA) have become common. A WTA contains a wheelchair lift and fastening devices. It enables wheelchair-bound persons to ride in vehicles, and therefore helps to extend their everyday activities. It is anticipated that such apparatuses will be used for long-distance travel in the near future. However, in the current design, riding comfort and minimizing user fatigue have not been adequately considered. Some users have actually indicated that the apparatus is uncomfortable. Therefore, a design that considers user comfort is required.

A sense of the riding comfort is a subjective evaluation, which mainly originates from the amount of vibration the user feels. To design a comfortable WTA, both vibration characteristics and user sensitivity need to be studied. In a previous study on vibration [1], we constructed a vibration simulation of a

Received March 31, 2000

Accepted November 20, 2000 passenger-wheelchair system, which can reflect the physical constitutions of passengers and types of wheelchairs. Thus, the objective of the present study is to examine the human sensitivity to vibration, and we have proposed a method to support designing a WTA by the findings of this study.

First, we carried out a comfort evaluation experiment with a vibration generator that vibrated the passenger-wheelchair system and measured floor vibration, vibration on the human body, and subjective evaluation. Secondly, based on the experimental results, we clarified the quantitative relationship between vibration and subjective evaluation. Finally, we proposed a vibration evaluation model, which is applied the findings obtained in this study.

\section{COMFORT EVALUATION EXPERIMENT}

\subsection{Measurement method}

Input vibration (floor vibration) was measured on a 


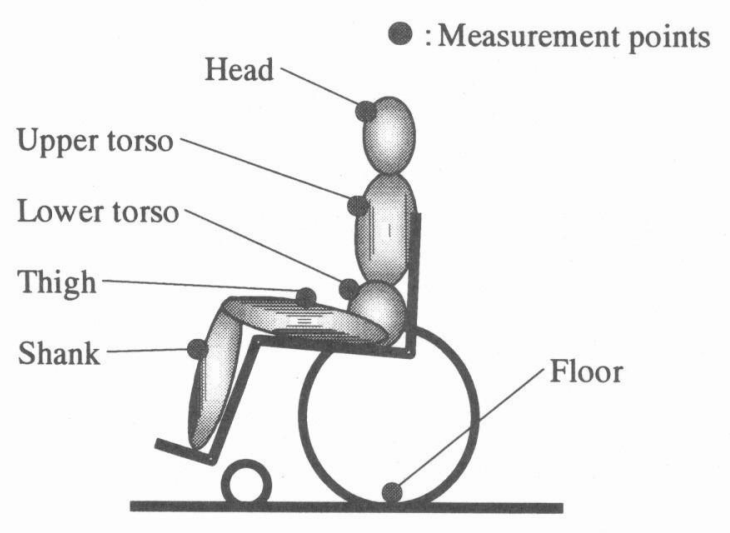

Figure 1: Measurement points where acceleration pick-up sensor were attached

vibration table at the midpoint between the two rear wheels of the wheelchair (Figure 1). Due to human joint rotation, the body of passenger was divided into five segments: head, upper torso, lower torso, thigh, and shank. The output vibration was measured at points on the surface of each body segment. These points represented the nearest points to the center of gravity that was estimated by Ae's study [2], which investigated the ratio of the center of gravity position to the length of each segment. The center of gravity of the wheelchair was obtained by hanging the wheelchair at two different points and drawing lines to find the cross point.

Piezoelectric acceleration pick-up sensors were placed on each measurement point in the vertical and horizontal directions, and vibration data was recorded on a data recorder through a charge amplifier.

We measured riding comfort in order to obtain a subjective evaluation for vibration. We measured the passengers' comfort and 8 types of vibration (Table 1), which were based on past studies [3,4]. To measure these items, we used the 5-level semantic differential method, which can easily quantify subjective evaluation.

\subsection{Experimental conditions}

The input vibration was set to vertical vibration. The frequency was set at 6 levels: $(2,4,6,8,10$, and $12 \mathrm{~Hz})$. According to Okada's study [5], people feel the most uncomfortable from vibration at $1-20 \mathrm{~Hz}$, and our preliminary experiment found that the frequency range could be limited to $12 \mathrm{~Hz}$.

The acceleration of input vibration was set at three levels: $0.41,0.63$, and $1.00 \mathrm{~m} / \mathrm{s}^{2}$ in view of the following data: the comfort decline curve boundary in minutes in ISO-2631 [6], the riding comfort limitative curve found by Janeway [7], and our previous study that found the
Table 1: Measurement items

\begin{tabular}{|c|c|}
\hline & $\leftarrow$ Level 5 \\
\hline Riding Comfort & Uncomfortable/Comfortable \\
\hline Sense of Vibration & 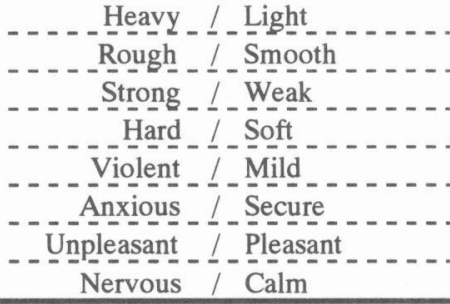 \\
\hline
\end{tabular}

average acceleration of the vibration on the floor of the running vehicle was $0.41 \mathrm{~m} / \mathrm{s}^{2}$ [8].

The examinees were seven Japanese young males. The wheelchair was a bi-manual rear-wheel-driven wheelchair, which currently comprises $70 \%$ of all wheelchairs used in Japan.

The measurement time was set at 2 minutes. 15 minutes interval was set between the measurements in order to for reducing the remaining mental effect of last measurement.

\section{EXPERIMENTAL RESULTS AND DISCUSSION}

\subsection{Vibration on the body}

Figure 2 shows the acceleration of the vibration transmitted to each segment of the body at each three levels of input acceleration. The acceleration of the vibration on the body was the square sum of the acceleration of the horizontal and vertical directions.

We can see from Figure 2 that the acceleration on each segment has a maximum value at a specific frequency, and that the frequency varies for each segment. It is likely that this is due to the system resonance. In addition, the acceleration on each segment increases with an increase in input acceleration.

\subsection{Riding comfort}

Figures 3 and 4 show the experimental results of riding comfort measurements. The relationship between riding comfort and input frequency is illustrated in Figure 3, and input acceleration is shown in Figure 4. The evaluation values are the average values for the seven examinees.

From these figures we can draw the following conclusions about riding comfort:

1. With an increase in frequency, the value of discomfort increases at $2-6 \mathrm{~Hz}$, peaks at 6 or $8 \mathrm{~Hz}$, and decreases at $8-12 \mathrm{~Hz}$. 


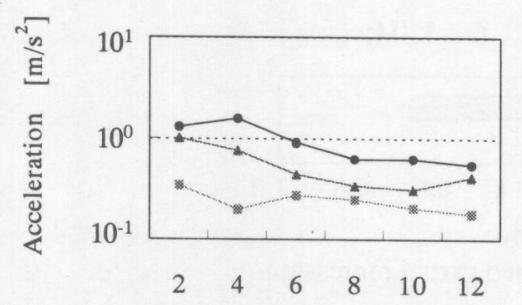

(a) Head

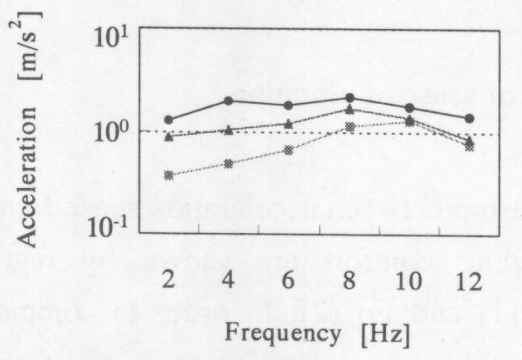

(d) Thigh

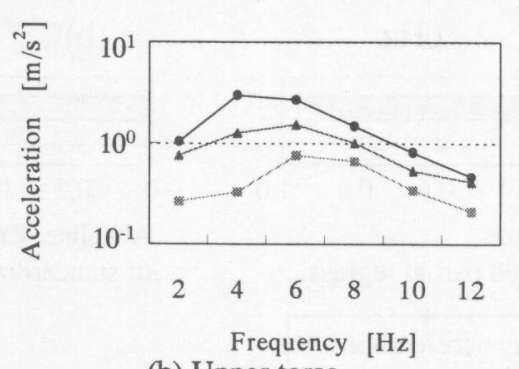

(b) Upper torso

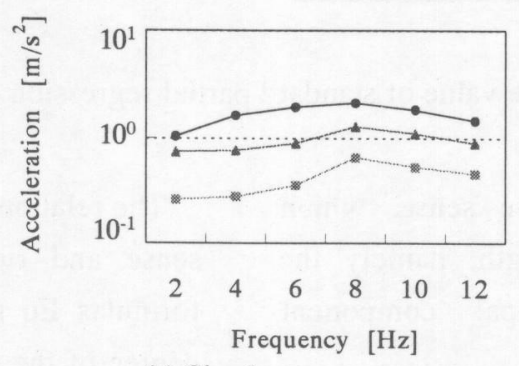

(e) Shank

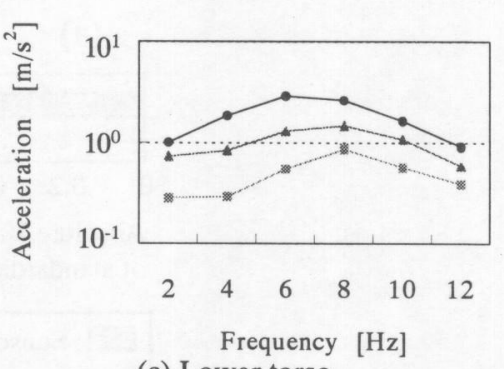

(c) Lower torso

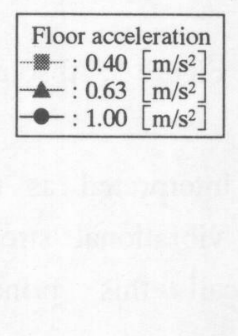

Figure 2: Vibration on the body

2. With an increase in frequency, the value of discomfort increases. However, 2-6 Hz clearly has a larger degree of discomfort than and 8-12 Hz.

From these two features, it can bee seen that there is a large difference between the input frequencies of 2-6 and 6-12 Hz. Therefore, we have divided the results into two sections for these two frequency ranges.

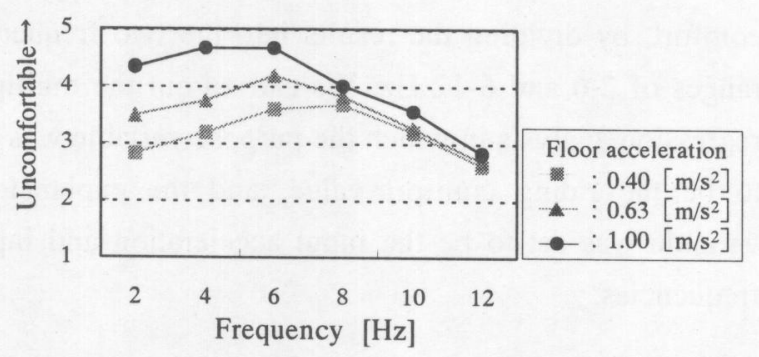

Figure 3: Relation between riding comfort and input frequency

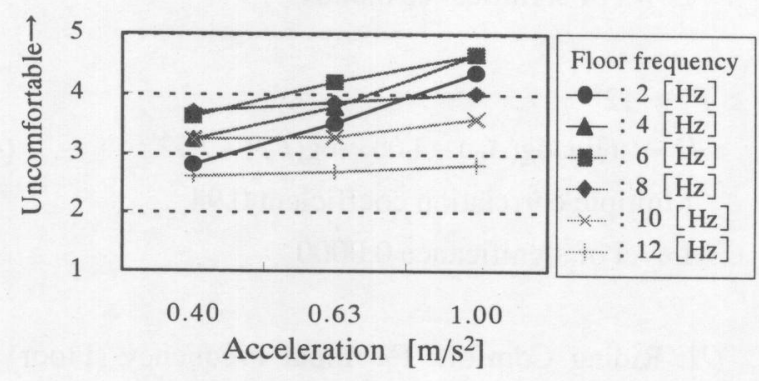

Figure 4: Relationship between riding comfort and input acceleration

\subsection{Sense of vibration}

Since the subordination relation was indicated between some measurement items, a principal component analysis was carried out. Factor load after the burr max rotation at each frequency range is shown in Tables 2 (a) and (b).

As these tables indicate, two common principal components exist for both frequency ranges. Factor loads of "strong", "violent", and "hard" are high for the first principal component. Therefore, the first principal

Table 2: Factor loads of principal component

$$
\text { (a) } 2 \sim 6 \mathrm{~Hz}
$$

\begin{tabular}{|c|c|c|}
\hline & $\begin{array}{c}\text { First principal component } \\
\text { (Acceleration sense) }\end{array}$ & $\begin{array}{c}\text { Second principal component } \\
\text { (Frequency sense) }\end{array}$ \\
\hline \hline Strong & 0.78 & 0.35 \\
\hline Violent & 0.79 & 0.31 \\
\hline Anxious & 0.84 & 0.21 \\
\hline Unpleasant & 0.84 & 0.02 \\
\hline Nervous & 0.86 & 0.08 \\
\hline Hard & 0.73 & 0.26 \\
\hline Rough & 0.04 & 0.87 \\
\hline Heavy & -0.08 & 0.87 \\
\hline \hline Ratio of contribution [\%] & 56.7 & 13.3 \\
\hline
\end{tabular}

\section{(b) $6 \sim 12 \mathrm{~Hz}$}

\begin{tabular}{|c|c|c|}
\hline & $\begin{array}{c}\text { First principal component } \\
\text { (Acceleration sense) }\end{array}$ & $\begin{array}{c}\text { Second principal component } \\
\text { (Frequency sense) }\end{array}$ \\
\hline \hline Strong & 0.80 & 0.22 \\
\hline Violent & 0.88 & -0.13 \\
\hline Anxious & 0.87 & 0.16 \\
\hline Unpleasant & 0.70 & 0.12 \\
\hline Nervous & 0.89 & 0.02 \\
\hline Hard & 0.86 & -0.07 \\
\hline Rough & 0.06 & 0.87 \\
\hline Heavy & 0.03 & 0.87 \\
\hline \hline Ratio of contribution [\%] & 53.1 & 19.4 \\
\hline
\end{tabular}


(a)

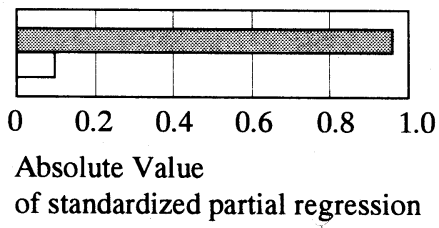

(b) $6-12 \mathrm{~Hz}$

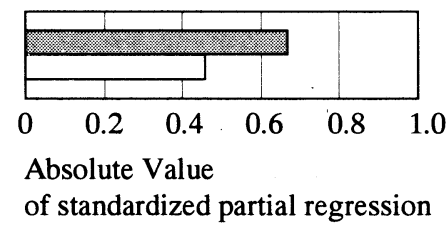

: Sensory acceleration

: Sensory frequency

Figure 5: Absolute value of standard partial regression of sense of vibration

component can be interpreted as the sense, which originates from the vibrational strength, namely the acceleration. We call this principal component "acceleration sense."

On the other hand, the factor loads of "rough" and "heavy" are high for the second principal component. Therefore, the second principal component can be interpreted as the sense, which originates from the period of vibration or the frequency. We call this principal component "frequency sense".

The values of acceleration sense and frequency sense are the values that evaluated the acceleration and frequency of the vibration. Therefore, in order to quantitatively examine the relationship between acceleration sense, frequency sense, and riding comfort, multiple regression analyses were carried out. The purpose variable was set to be the riding comfort value, and the explanatory variable was set to be the acceleration sense and frequency sense. As a result, significant regression formulas were obtained as follows.

$2 \leq F_{I} \leq 6$

$H=0.73 \times P C S_{A}-0.09 \times P C S_{F}+3.92$

Multiple correlation coefficient 0.95 ,

Level of significance 0.0007

$6 \leq F_{I} \leq 12$

$H=0.49 \times P C S_{A}+0.59 \times P C S_{F}+3.50$

Multiple correlation coefficient 0.95 ,

Level of significance 0.0003

( $H$ : Riding Comfort, $F_{I}$ :Input Frequency (Floor)

$P C S_{A}$ : Principal Component Score of Acceleration Sense

$P C S_{F}$ : Principal Component Score of Frequency Sense)
The relationship between acceleration sense/frequency sense and riding comfort are shown by regression formulas $\mathrm{Eq}$ (1) and $\mathrm{Eq}$ (2). In order to compare the degree of the effect of acceleration sense and frequency sense on riding comfort, absolute values of standardized partial regression of acceleration sense and frequency sense in each regression formula are illustrated in Figure 5. This figure tells us that at $2-6 \mathrm{~Hz}$, the effect of acceleration sense for the riding comfort is significant, and that at 6-12 $\mathrm{Hz}$, both the acceleration sense and frequency sense greatly influence riding comfort.

\subsection{Relationship between floor vibration and riding comfort}

Judging from the previous sections, it is possible to quantify the relationship between input acceleration/frequency of floor vibration, and riding comfort, by dividing the results into the two frequency ranges of 2-6 and 6-12 $\mathrm{Hz}$. We carried out the multiple regression analysis in which the purpose variable was set to be the riding comfort value, and the explanatory variable was set to be the input acceleration and input frequencies.

$$
\begin{aligned}
2 \leq & F_{I} \leq 6 \\
& H=1.93 \times \log \left(A_{F}\right)+1.77 \times \log \left(F_{F}\right)+2.98 \\
& \text { Multiple correlation coefficient } 0.95, \\
& \text { Level of significance } 0.0007
\end{aligned}
$$

$$
\begin{aligned}
6 \leq & F_{I} \leq 12 \\
& H=1.60 \times \log \left(A_{F}\right)-4.08 \times \log \left(F_{F}\right)+7.53 \\
& \text { Multiple correlation coefficient } 0.94 \\
& \text { Level of significance } 0.0000
\end{aligned}
$$

( $H$ : Riding Comfort, $F_{F}$ :Input Frequency (Floor), $F_{F}$ :Input Acceleration (Floor)) 
Using the regression formulas $\mathrm{Eq}(3)$ and $\mathrm{Eq}(4)$, it is possible to estimate the riding comfort from the floor vibration. In addition, by substituting the 5-level riding comfort value to $H$ in these regression formulas, lines which show the relationship between input acceleration and input frequency at each riding comfort value $H$ were calculated and shown in Figure 6. These formulas and figures will be useful riding comfort criteria when taking floor vibration in to consideration in the design of a WTA.

\subsection{Relationship between body vibration and riding comfort}

\subsubsection{Vibration on different body segments}

Next, the relationship between body vibration and riding comfort was examined by using correlation diagrams and correlation analysis. For the input frequency ranges $2-6$ and $6-12 \mathrm{~Hz}$, correlation diagrams of vibration acceleration on each body segment and

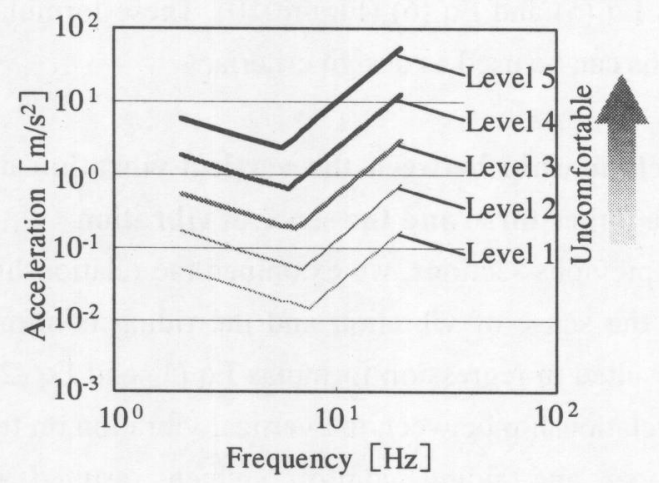

Figure 6: Floor vibration criteria riding comfort are shown in Figure 7. Straight line, which regressed the riding comfort by the acceleration of each segment, the regression formula and $t$ value were presented together in the correlation diagrams. In addition, the correlation coefficient for acceleration on each body segment and riding comfort are shown in Figure 8.

Figures 7 and 8 , at 2-6 Hz, confirm the correlation between the acceleration of the segments (except for the head) and riding comfort, because the correlation coefficients are $0.80-0.89$. On the other hand, at $6-12 \mathrm{~Hz}$, the correlation between the acceleration of the upper/lower torso and riding comfort is confirmed, because the correlation coefficients are 0.79-0.90. However, for the other segments, the correlation coefficients are $0.48-0.56$, which means the vibration on those segments does not greatly affect riding comfort.

Among the five body parts, only the acceleration of the upper and lower torsos have high correlation to the riding comfort at both input frequency range. The correlation coefficient of the upper torso is especially high. The main reason for this fact is thought to be that the respiratory system is included in the upper torso. It is reported that the resonant frequency of the respiratory system is 4-6 Hz [9]. Therefore, the upper torso vibration causes a feeling of suffocation in the person that increases the level of discomfort. Thus, we limited the subject of analysis to the upper torso in the following sections.

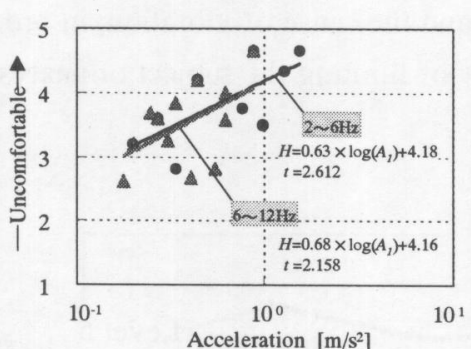

(a) Head

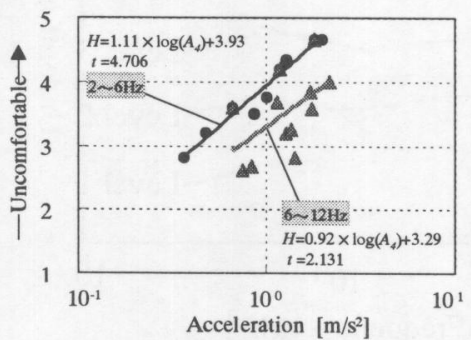

(d) Thigh

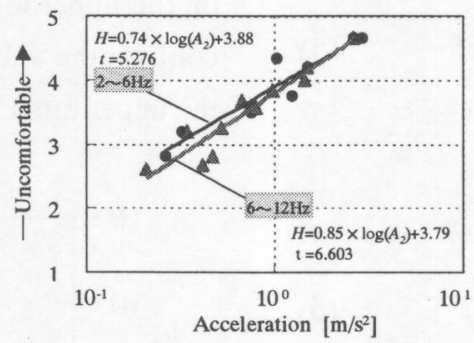

(b) Upper torso

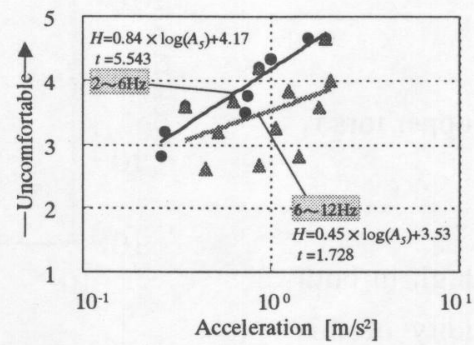

(e) Shank

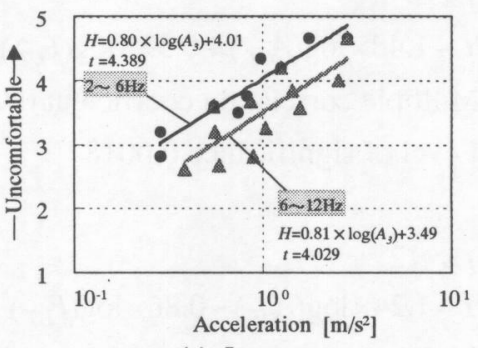

(c) Lower torso

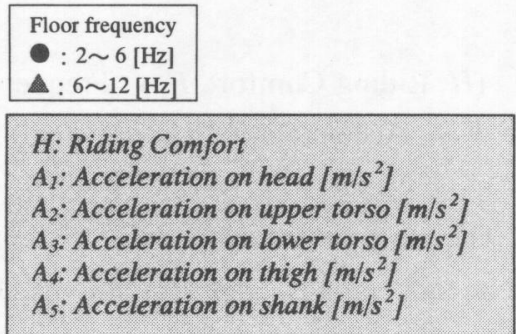

Figure 7: Correlation diagram of body vibration and riding comfort 


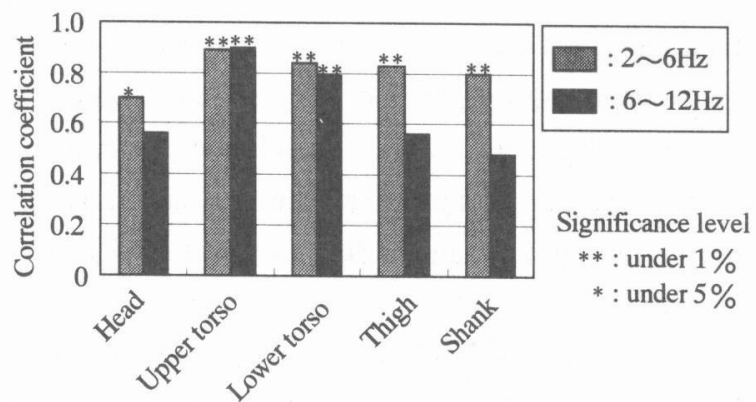

Figure 8: Correlation coefficient

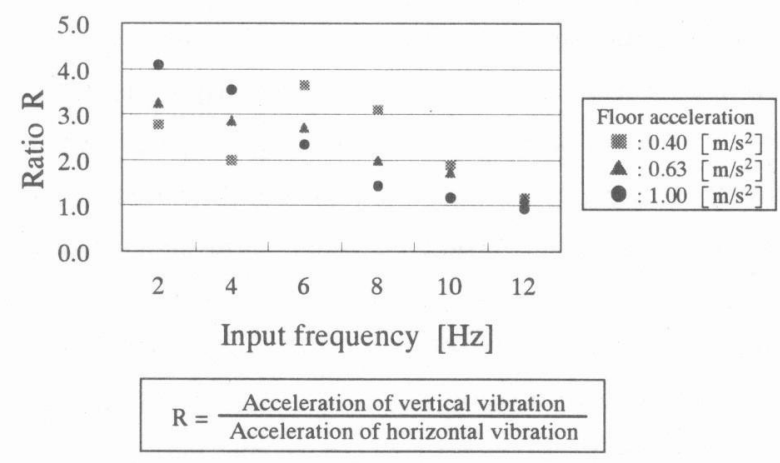

Figure 9: Ratio of vertical and horizontal acceleration on upper torso

\subsubsection{Effect of vibration directions}

We compared the difference between vertical and horizontal acceleration on the upper torso. The ratio of acceleration of both directions at each frequency was calculated, as shown in Figure 9. We see from the figure that the ratio is higher, namely vertical vibration is stronger than lower frequency, which causes more discomfort. Thus, in the next section we analyze the relationship between the vertical acceleration on the upper torso and riding comfort.

\subsubsection{Relationship between the vertical acceleration on the upper torso and the riding comfort}

We carried out multiple regression analyses in which the purpose variable was set to be the riding comfort value, and the explanatory variable was set to be the vertical acceleration and frequency on the upper torso.

$2 \leq F_{I} \leq 6$

$$
H=1.48 \times \log \left(A_{U T}\right)+0.53 \times \log \left(F_{U T}\right)+3.55
$$

Multiple correlation coefficient 0.98 ,

Level of significance 0.0018

$$
\begin{aligned}
6 \leq & F_{I} \leq 12 \\
& H=1.24 \times \log \left(A_{U T}\right)-0.86 \times \log \left(F_{U T}\right)+4.62 \\
& \text { Multiple correlation coefficient } 0.95, \\
& \text { Level of significance } 0.0002
\end{aligned}
$$

( $H$ : Riding Comfort, $F_{U T}$ : Frequency on upper torso, $F_{U T}$ : Acceleration on upper torso)

The multiple correlation coefficients are high in both frequency ranges, and that proves the validity of this regression formula.

These regression formulas enable us to estimate riding

comfort value from the vertical vibration on the upper torso. As well as in the case of the floor vibration, the line, which shows the relationship between the acceleration and frequency on the upper torso at each riding comfort value was deduced from the regression formulas Eq (5) and Eq (6) (Figure 10). These formulas and graphs can be used as design criteria.

\subsubsection{Relationship between the vertical vibration on the upper torso and the sense of vibration}

In the previous sections, we examined the relationship between the sense of vibration and the riding comfort, which resulted in regression formulas Eq (1) and Eq (2), and the relationship between the vertical vibration on the upper torso and riding comfort, which resulted in regression formulas $\mathrm{Eq}(5)$ and $\mathrm{Eq}(6)$.

In this section, we shall discuss the vertical vibration on the upper torso and the sense of vibration, in order to confirm the validity of limiting the subject of analysis to the upper torso.

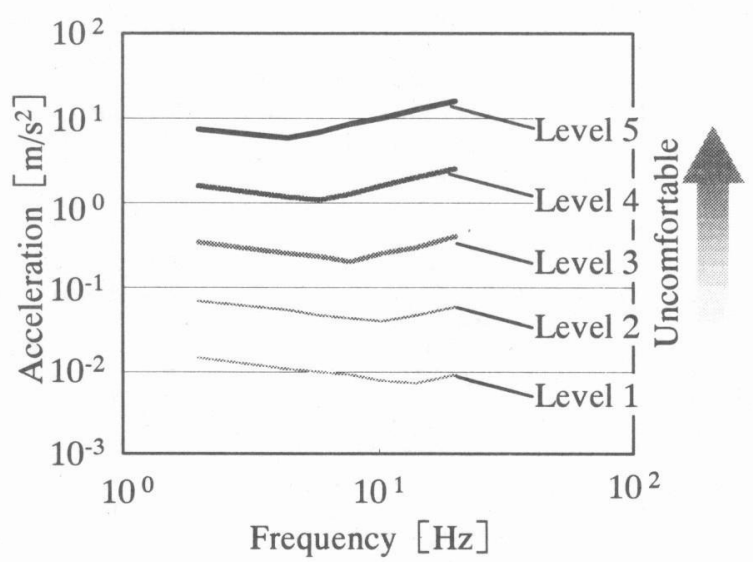

Figure 10: Body vibration criteria 
(a) $2 \sim 6 \mathrm{~Hz}$

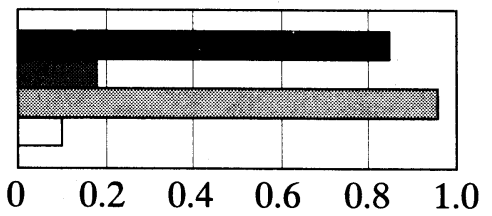

Absolute value of standardized partial regression (b) $6 \sim 12 \mathrm{~Hz}$

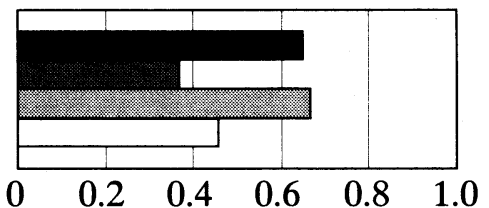

Absolute value of standardized partial regression

: Acceleration of vertical vibration on upper torso

: Frequency of vertical vibration on upper torso

: Sensory acceleration

Sensory frequency

Figure 11: Absolute value of standardized partial regression to riding comfort

Figure 11 shows the absolute values of standardized partial regression coefficient in the four regression formulas: (Eq (1),(2),(5) and (6)). The coefficients are equivalent between acceleration on the upper torso and acceleration sense, and frequency on the upper torso and frequency sense, that is to say the vibration sense corresponds to the vertical vibration on the upper torso. This fact proves the validity of the method to predict the riding comfort from the vertical vibration on the upper torso.

\section{METHOD OF APPLICATION TO THE DESIGN OF A WTA}

From the findings obtained above, we propose a vibration evaluation model (Figure 12). This model will enable us to estimate the riding comfort value from the vertical vibration on the upper torso. Therefore, this value, as well as the differences of passengers and the wheel chairs themselves, can be incorporated into a WTA design. However, to utilize these criteria, a model of the passenger-wheelchair system that can simulate the transmission of vibration from floor to the upper torso is also needed.

Most existing evaluation prediction methods use the floor or street vibration for their analysis. However, these methods cannot be used for systems that contain changing parameters, such as different passengers and types of wheelchairs.

In the past study [1], we constructed the vibration

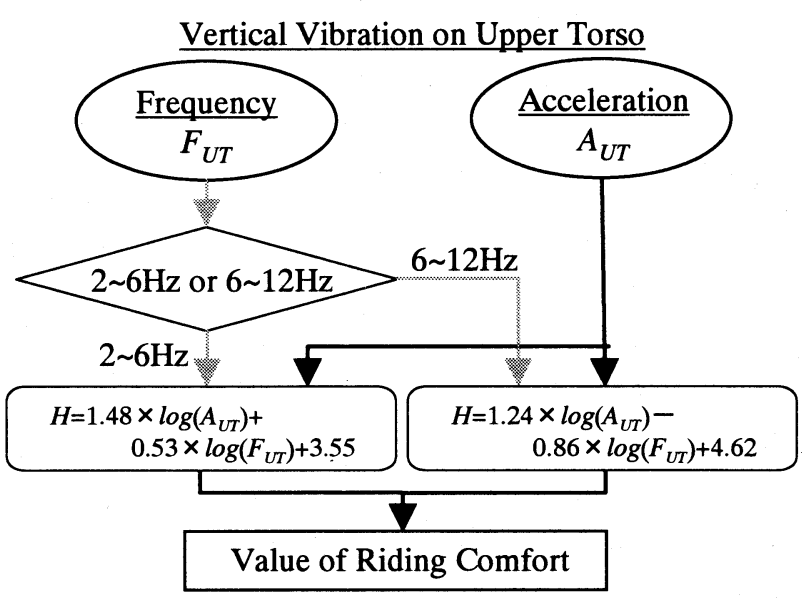

Figure 12: Vibration evaluation model

simulation model of the passenger-wheelchair system, which can reflect the difference of passengers and wheelchairs. When this vibration-transmission model and the vibration evaluation model are integrated, this model will be usable to improve the practical design of a WTA.

\section{CONCLUSION}

In this study, the quantitative relationship between subjective evaluation and vibration in a passenger-wheelchair system was clarified. A vibration evaluation model, which enabled us to predict the riding comfort value from the vertical vibration on the upper torso was also constructed.

In addition, two criteria: floor vibration and body vibration were proposed. The former is valuable when 
only consideration for the vibration on the floor is required. The latter is valuable when reflection of physical constitution of passengers or types of wheelchairs is required.

In the future, we will construct a comfort-prediction model that consists of a vibration-evaluation model and vibration-transmission model. Moreover, in order to improve the model to further WTA design, we plan study the physical and mental characteristics senior citizens using wheelchairs, since they are the main users of wheelchairs in Japan.

\section{Acknowledgments}

The authors would like to thanks: Mr. Masao Okajima, Mr. Isao Takeda, Mr. Isao Hayashi and Mr. Masatsugu Saito of Ikeda Bussan Co., LTD., for the help and generosity in the completion of this research.

\section{REFERENCES}

1. K. Kawai, H. Yonekawa, eds.; A Vibration Simulation Model of Human Body-Wheelchair System on the Wheelchair Transporting Apparatus, The Science of Design (1999)

2. M . Ae, et al.; Estimation of Inertia Properties of the Body Segment in Japanese Athletes, Journal of Bio-mechanism, 124, 23-33 (1992)

3. K. Ryu; The basic research on vibration response of human body, Doctoral Dissertation, Chiba University (1996)

4. H. Kanda, S. Nanba; Human sensibility evaluation to ship vibration by semantic differential method, Human Engineering, 10, 2, 55-62 (1974)

5. A. Okada; Sense of Vibration of Man, Journal of Society of Automotive Engineers of Japan 34-5, 440-450 (1980)

6. ISO-2631; Guide for the Evaluation of Human Exposure to Wholebody Vibration

7. Janeway, R. N.; Vehicle vibration limit to fit the passenger, SAE journal 48 (1948)

8. H. Yonekawa, Y. Matsuoka; Vibration Analysis and Design Problem of the Transport System by Vehicle with Persons Seated in Wheelchairs, The Third Asia Design Conference 1, 169-176 (1998)

9. A. Okada, et al.; Effect of Noise, vibration, and impact, Human and Technology, 276-28 (1970) 